\title{
Erratum
}

\section{Erratum to: Chinese Journal of Mechanical Engineering}

\section{Erratum to: Transient-spatial pattern mining of eddy current pulsed thermography using wavelet transform}

DOI: 10.3901/CJME.2014.0421.079

\begin{abstract}
YANG Hailong ${ }^{1}$, GAO Bin ${ }^{1, *}$, TIAN Guiyun ${ }^{1,2}$, REN Wenwei ${ }^{1}$, and WOO Wai Lok $^{2}$
1 School of Automation Engineering, University of Electronic Science and Technology of China, Chengdu 611731, China 2 School of Electrical and Electronic Engineering, Newcastle University, UK
\end{abstract}

\begin{abstract}
The original version of this article unfortunately contained a mistake. The presentation of the DOI in printed version was incorrect. The corrected DOI is given below.
\end{abstract}

DOI: 10.3901/CJME.2014.0526.100

* Corresponding author. E-mail: bin_gao@uestc.edu.cn

Supported by National Natural Science Foundation of China(Grant No. 51377015), China Post Doctor Project(Grant No. 136413), and Science \& Technology Department of Sichuan Pr ovince, China(Grant No. 2013HH0059) 